\title{
Effects of Insall-Salvati ratio and obesity on suprapatellar fat pad
}

\author{
Mehmet ŞiRiK ${ }^{1}$, Ibrahim INAN² \\ ${ }^{1}$ Department of Radiology, Faculty of Medicine, Adiyaman University, Adiyaman, Turkey \\ ${ }^{2}$ Department of Radiology, Biruni University Hospital, İstanbul, Turkey \\ Correspondence \\ Mehmet Şíik \\ Adıyaman Üniversitesi Tıp Fakültesi Eğitim ve Araştırma Hastanesi Adıyaman Üniversitesi Tıp Fakültesi Radyoloji Anabilim Dalı 02100 Adıyaman, Türkiye \\ e-mail:dr.mmtsrk@gmail.com
}

\section{ABSTRACT}

This study aimed to evaluate the effect of Insall-Salvati ratio and obesity on signal and shape changes in the suprapatellar fat pad and investigate their role in impingement.

A total of 124 patients who underwent magnetic resonance imaging examination of the knee between January and November 2018 were included in the study. The ratio of the length of the patellar tendon to patella was calculated for the InsallSalvati ratio. The thickness of subcutaneous fat tissue was measured from the medial of the knee as an indicator of obesity. The relationship between suprapatellar fat pad signal and shape changes, Insall-Salvati ratio, subcutaneous fat tissue thickness, quadriceps and patellar tendon thickness, and demographic data were statistically analyzed.

Of the patients, 59 (47.6\%) were women and 65 (52.4\%) were men. In 56 (45.2\%) patients, the suprapatellar fat pad had signal changes compatible with those of edema. Moreover, 77 (62.1\%) patients had a mass effect on the adjacent joint space due to convexity in the suprapatellar fat pad posterior contour. Although the mass effect was found in $63.6 \%(49)$ of the patients with edema in the suprapatellar fat pad, it was missing in $85.1 \%$ (40) of the patients without edema in the suprapatellar fat pad $(P<0.001)$.

This study showed that although a significant relationship existed between the presence of edema and mass effect in the suprapatellar fat pad, the Insall-Salvati ratio and obesity had no significant effect on suprapatellar fat pad impingement.

Key words: Impingement, Insall-Salvati ratio, suprapatellar fat pad

\section{INTRODUCTION}

Anterior knee pain is an important part of orthopedic complaints, and most of the musculoskeletal magnetic resonance imaging (MRI) examinations are performed due to these symptoms. The ligaments, retinaculum, and bone structures are passive stabilizers of the patellofemoral compartment, whereas quadriceps muscle movement is an active stabilizer. Traumatic, congenital, or atrophic imbalances in this system can cause impingement in the surrounding tissue and patellar chondromalacia, leading to anterior knee pain (1). The fat pads in the knee have intense innervation and vascularization and are highly sensitive (2). Patellar localization abnormalities are known to be associated with knee pain. The upwardly placed patella (patella alta) is important in the development of chondromalacia. Furthermore, patellofemoral imbalance increases the risk of patellar subluxation or dislocation (3). The most commonly used method for evaluating patellar placement is the ratio of patellar tendon length to patellar length (TL/PL), which is known as the Insall-Salvati ratio (3). Obesity is known to contribute to many degenerative pathologies such as osteoarthritis in the knee joint (4). This study aimed to investigate the effects of obesity and InsallSalvati ratio on the development of impingement in the suprapatellar fat pad.

\section{MATERIALS AND METHODS}

This study was conducted retrospectively and approved by the local ethics committee (2018/7-18). A total of 124 patients were included in the study; 59 (47.6\%) patients were women and 65 (52.4\%) patients were men. The median age of the patients was 26 years; the minimum age was 18 years and the maximum was 
Table 1: Standard protocol for knee MRI.

\begin{tabular}{|c|c|c|c|c|c|}
\hline & Plane & $\begin{array}{l}\text { Repetition time }(\mathrm{TR}) \text {, } \\
\mathrm{ms}\end{array}$ & $\begin{array}{l}\text { Echo time (TE), } \\
\text { ms }\end{array}$ & $\begin{array}{l}\text { Slice thickness, } \\
\mathrm{mm}\end{array}$ & Gap, mm \\
\hline \multirow[t]{3}{*}{ SPAIR sequences } & Sagittal & 3034 & 30 & 3.5 & 0.3 \\
\hline & Coronal & 3034 & 30 & 3.5 & 0.3 \\
\hline & Axial & 3034 & 30 & 3.5 & 0.3 \\
\hline T1W TSE (turbo spin-echo) & Sagittal & 560 & 17 & 3.5 & 0.6 \\
\hline
\end{tabular}

51 years. Patients older than 18 years were included in the study, and the patients who had undergone trauma or knee operation were excluded. A total of 124 randomly selected patients who underwent knee MRI examination between January and November 2018 were enrolled in the study. All MRI examinations were performed using a 1.5T Achieva Scanner (Philips, Netherlands) with a standard protocol for the knee examination consisting of four sequences (Table 1).

A radiologist with 15 years of experience undertook the evaluation of MRIs using sagittal plane T1weighted, fat-suppressed axial, and sagittal plane T2weighted images. If the fat signal on fat-suppressed T2-weighted images was greater than the muscle signal and less than the fluid signal, it was defined as edema on the fat pad (Fig. 1). The TL/PL was calculated for the Insall-Salvati ratio (Fig. 2). The thickness of subcutaneous fat tissue was measured from the medial of the knee in all patients as an indicator of obesity using MRIs in the axial plane (Fig. 3). The relationships between suprapatellar fat pad signal and shape changes, Insall-Salvati ratio, subcutaneous fat tissue thickness, quadriceps and patellar tendon thickness, and demographic data were statistically analyzed.

The SPSS version 21.0 (IBM Corporation, NY, USA) was used for statistical analysis. Categorical data were presented as numbers and percentages, and numerical data were expressed as means and standard deviations. If the numerical data did not show a normal distribution, the median, minimum, and maximum values were used instead. The Shapiro-Wilk test was used to evaluate the normal distribution of numerical data. The Mann-Whitney $U$ and Kruskal-Wallis test were used to compare differences between groups in terms of nonparametric data, and the categorical data were compared between groups using Pearson's chi-squared tests.

\section{RESULTS}

Signal changes in the suprapatellar fat pad compatible with edema were found in 56 patients (45.2\%). In $68(54.8 \%)$ cases, the suprapatellar fat pad signal was normal. In 77 (62.1\%) patients, a mass effect was observed on the adjacent joint space due to convexity in the suprapatellar fat pad posterior contour. Although the mass effect was found in $63.6 \%$ (49) of the patients with edema in the suprapatellar fat pad, it was missing in $85.1 \%$ (40) of the patients without edema in the suprapatellar fat pad $(P<0.001)$ (Table 2$)$. No statistically significant difference was found in Insall-Salvati ratio and subcutaneous adipose tissue thickness with the presence of edema in the suprapatellar fat pad $(P=$ 0.874 and 0.448 , respectively). Similarly, when the age,

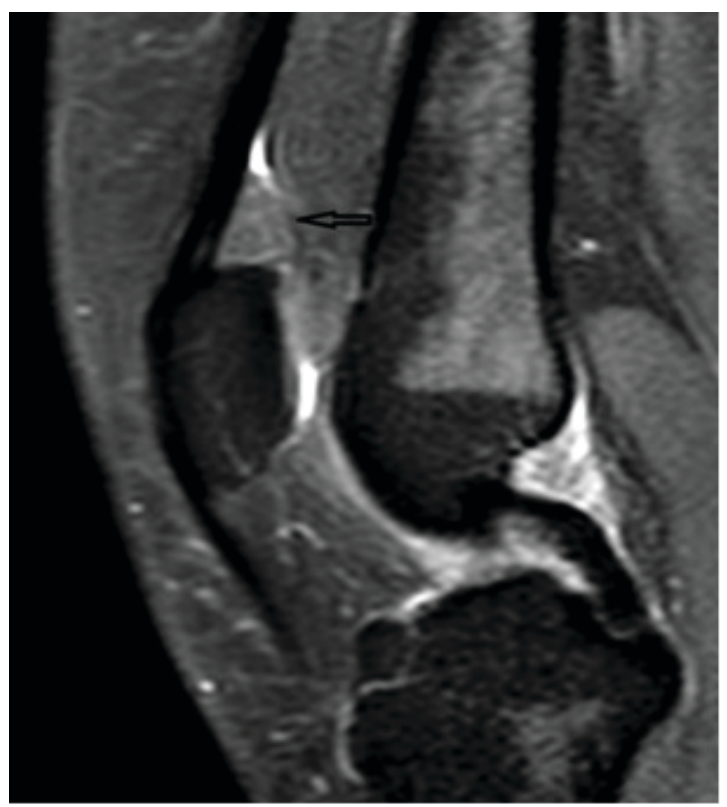

Figure 1: Edema in the suprapatellar fat pad on fat-suppressed T2-weighted images. 


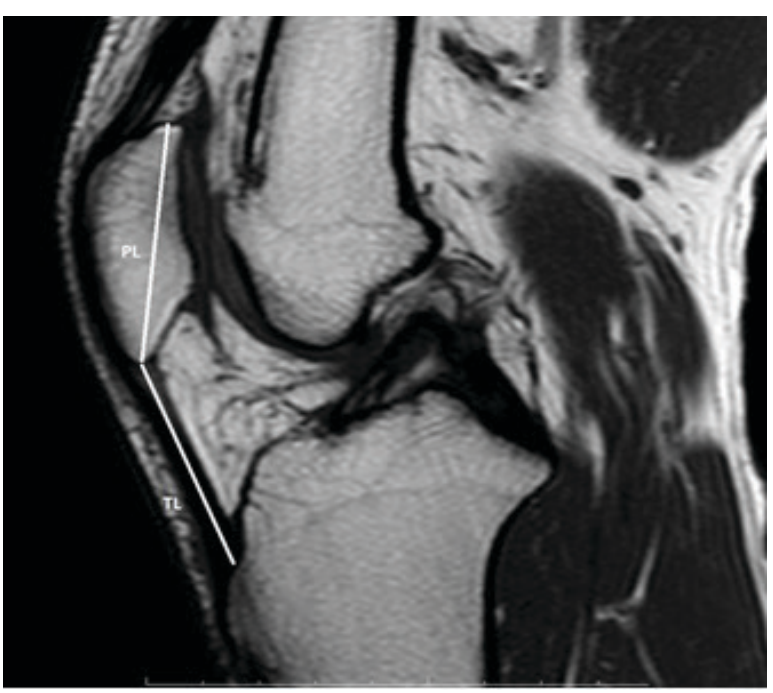

Figure 2: Ratio of the length of the patellar tendon to the length of the patella (TL/PL) was calculated for the InsallSalvati ratio.

Table 2: Relationship of edema in the suprapatellar fat pad with mass effect.

\begin{tabular}{lcc}
\hline & \multicolumn{2}{c}{ Mass effect } \\
\cline { 2 - 3 } & Found & Not found \\
\hline Suprapatellar fat pad & $49(63.6 \%)$ & $7(14.9 \%)$ \\
Edema & $28(36.4 \%)$ & $40(85.1 \%)$ \\
\hline
\end{tabular}

quadriceps, and patellar tendon thickness and length were evaluated with the presence of edema in the suprapatellar fat pad, no statistically significant relationship was found (Table 3).

\section{DISCUSSION}

Similar pathological changes may occur in the infrapatellar and suprapatellar fat pad pathologies in the anterior compartment of the knee. However, signal changes in the suprapatellar fat pad may be overlooked in daily practice compared with the infrapatellar fat pad. The infrapatellar fat pad expansion and edema due to trauma and impingement were defined in Hoffa's disease, and the changes in the suprapatellar fat pad were thought to have similar mechanisms (5). The pathological processes related to these changes cause anterior knee pain. Anterior knee pain from the patellofemoral compartment is a common clinical symptom in young people (6). The subjective evalu-

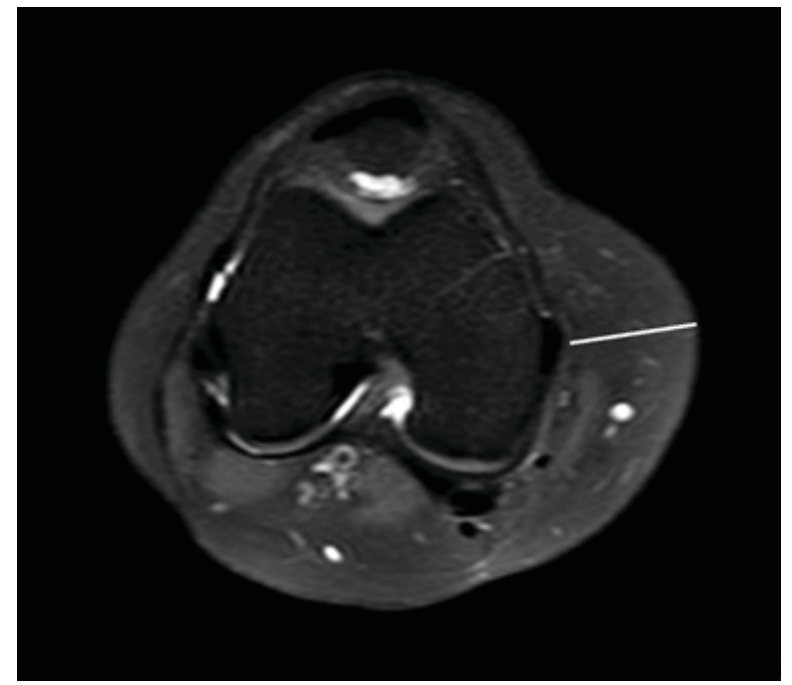

Figure 3: Thickness of subcutaneous fat tissue was measured from the medial of the knee in all patients as an indicator of obesity via MRIs in the axial plane.

ation of the posterior surface of the fat pad for convexity is referred to as the most effective diagnostic method of suprapatellar fat pad expansion, and this increase in convexity is associated with anterior knee pain (7). Roth et al. showed that the prevalence of the enlargement of suprapatellar fat pad was found to be $12 \%$. This study reported a significant relationship between the fat pad signal and anterior knee pain (7) as well as between the mass effect due to enlargement in the suprapatellar fat pad and the fat signal. Wang et al. reported that the shape and signal changes in the suprapatellar fat pad were associated with knee pain (8). Consistent with this finding, the present study found a significant relationship between suprapatellar fat pad signal change and mass effect. Shabshin et al. reported that suprapatellar fat pad edema might be similar to Hoffa's disease, and could be a cause of anterior knee pain, but its association with symptoms was unclear (9).

A previous study showed a significant relationship between Insall-Salvati ratio and anterior cruciate ligament $(A C L)$ tears, and the patella alta variation increased the risk of ACL tear (10). In another similar study, a positive correlation was found between ACL tears and patella baja (11). Gursoy et al. reported a significant relationship between infrapatellar fat pad edema and chondromalacia patella with patellar maltracking (12). Similarly, Jibri et al. demonstrated 
Table 3: Relationship between edema in the suprapatellar fat pad and numerical data.

\begin{tabular}{llll}
\hline & \multicolumn{2}{c}{ Edema in the suprapatellar fat pad } & P value \\
\cline { 2 - 3 } & Found & Not found & 0.654 \\
\hline Patellar tendon length & $43.76 \pm 6.6$ & $44.31 \pm 6.75$ & 0.46 \\
Length of patella & $40.46 \pm 4.25$ & $41.03 \pm 4.17$ & 0.875 \\
Quadriceps tendon thickness & $7.82 \pm 1.13$ & $7.78 \pm 1.24$ & 0.727 \\
Patellar tendon thickness & $4.44 \pm 0.58$ & $4.4 \pm 0.7$ & 0.298 \\
Age & $26(18-44)$ & $24.5(18-51)$ & 0.874 \\
Insall-Salvati ratio & $1.05(0.75-1.46)$ & $1.05(0.73-1.52)$ & 0.75 \\
Qaudriceps/Patellar tendon thickness ratio & $1.72(1.32-2.56)$ & $1.76(0.98-3.13)$ & 0.448 \\
Subcutaneous fat tissue thickness & $19.1(8.5-38.5)$ & $19.65(7.2-37.6)$ & \\
\hline
\end{tabular}

that edema in the superolateral portion of Hoffa's fat pad was associated with patellar maltracking (13). In the present study, no significant relationship was found between the Insall-Salvati ratio and suprapatellar fat pad edema.

Kok et al., using MRI, reported that the subcutaneous knee fat thickness as a surrogate marker of obesity was positively associated with the presence and severity of chondromalacia patella (14). In this study, subcutaneous knee fat thickness did not have a significant effect on the development of impingement in the suprapatellar fat pad.

\section{REFERENCES}

1. Chung CB, Skaf A, Roger B, Campos J, Stump X, Resnick D. Patellar tendon-lateral femoral condyle friction syndrome: MR imaging in 42 patients. Skeletal Radiol. 2001;30(12):694-697.

2. Borja MJ, Jose J, Vecchione D, Clifford PD, Lesniak BP. Prefemoral fat pad impingement syndrome: identification and diagnosis. American journal of orthopedics (Belle Mead, NJ), (2013); 42(1), E9-11.

3. Shabshin N, Schweitzer ME, Morrison WB, Parker L. MRI criteria for patella alta and baja. Skeletal radiology, (2004); 33(8), 445450.

4. Coggon D, Reading I, Croft P, McLaren M, Barrett, D, Cooper C. Knee osteoarthritis and obesity. International journal of obesity, (2001); 25(5), 622.

5. Jacobson JA, Lenchik L, Ruhoy MK, Schweitzer ME, Resnick D. MR imaging of the infrapatellar fat pad of Hoffa. RadioGraphics 1997; 17:675-691.

6. Jackson AM. Anterior knee pain. J Bone Joint Surg $\mathrm{Br}$ 2001;83:937e48.

7. Roth C, Jacobson J, Jamadar D, Caoili E, Morag Y, Housner J. Quadriceps fat pad signal intensity and enlargement on MRI: prevalence and associated findings. American Journal of Roentgenology, (2004); 182(6), 1383-1387.

8. Wang J, Han W, Wang X, Pan F, Liu Z, Halliday A, et al. Mass effect and signal intensity alteration in the suprapatellar fat pad: associations with knee symptoms and structure. Osteoarthritis and cartilage, (2014); 22(10), 1619-1626.
The limitations of this study were the retrospective nature of the study and the inability to directly examine patients with anterior knee pain. A largesample prospective study on patients with anterior knee pain may provide more meaningful results.

\section{CONCLUSIONS}

This study showed that the signal changes in the suprapatellar fat pad were compatible with those of edema, and the presence of mass effect was significantly associated with it. This relationship might play an important role in anterior knee pain.

9. Shabshin N, Schweitzer ME, Morrison WB. Quadriceps fat pad edema: significance on magnetic resonance images of the knee. Skeletal radiology, (2006); 35(5), 269-274.

10. Degnan AJ, Maldjian C, Adam RJ, Fu FH, Di Domenica M. Comparison of Insall-Salvati ratios in children with an acute anterior cruciate ligament tear and a matched control population. American Journal of Roentgenology, (2015); 204(1), 161-166.

11. Lin CF, Wu JJ, Chen TS, Huang TF. Comparison of the InsallSalvati ratio of the patella in patients with and without an $\mathrm{ACL}$ tear. Knee Surg Sports Traumatol Arthrosc 2005; 13:8-11.

12. Gürsoy $M$, Mete BD, Oyar $O$, Erdoğan N, Uluç ME, Bulut $T$, et al. The association of patellar maltracking with infrapatellar fat pad edema and chondromalacia patella: A quantitative morphological magnetic resonance imaging analysis. Turk J Phys Med Rehab., 2018;64(3):246-252

13. Jibri Z, Martin D, Mansour R, Kamath $S$. The association of infrapatellar fat pad oedema with patellar maltracking: a casecontrol study. Skeletal Radiol 2012; 41:925-31.

14. Kok HK, Donnellan J, Ryan D. Torreggiani, W. C. Correlation between subcutaneous knee fat thickness and chondromalacia patellae on magnetic resonance imaging of the knee. Canadian Association of Radiologists Journal, (2013); 64(3), 182-186. 